\title{
A SIMPLE PROOF OF THE KÜNNETH THEOREM
}

\section{ALEX HELLER}

The following proof of a much-proved theorem is offered in the belief that it is simpler than the many existing ones (cf. for example $[1 ; 2, \S 12])$. It has also the virtue of a form suitable to generalization to abstract categories, which will be undertaken in another place [4].

By a chain complex we shall mean a graded abelian group with a derivation of degree -1 . A chain complex $X$ is positive if $X_{i}=0$ for $i<0$. If $X$ and $Y$ are positive chain complexes then so is $X \otimes Y$. If at least one of $X, Y$ is a free abelian group then the Künneth theorem relates the homology of $X \otimes Y$ to the homology of $X$ and $Y$.

Recall that for abelian groups $A, B$ graded by non-negative degrees $(A \otimes B)_{n}=\sum_{i+j=n} A_{i} \otimes B_{j}$. We write also

$$
\operatorname{Tor}_{1}(A, B)_{n}=\sum_{i+j=n} \operatorname{Tor}_{1}\left(A_{i}, B_{j}\right)
$$

and

$$
\operatorname{Ext}^{1}(A, B)_{n}=\sum_{i+j=n} \operatorname{Ext}^{1}\left(A_{i}, B_{j}\right),
$$

so that $\operatorname{Tor}_{1}(A, B)$ and $\operatorname{Ext}^{1}(A, B)$ are graded groups.

If $X$ and $Y$ are positive chain complexes then $X \otimes Y$ has the derivation given by $\partial(x \otimes y)=\partial x \otimes y+(-1)^{i} x \otimes \partial y$ on $X_{i} \otimes Y_{j}$.

TheOREM 1 (KüNNETH). If $X$ and $Y$ are positive chain complexes then there is an exact sequence

$$
0 \rightarrow H X \otimes H Y \stackrel{\mathfrak{S}}{\rightarrow} H(X \otimes Y) \stackrel{\mathfrak{D}}{\rightarrow} \operatorname{Tor}_{1}(H X, H Y) \rightarrow 0,
$$

the maps $\mathfrak{S}, \mathfrak{D}$ being homogeneous of degrees $0,-1$.

We begin by considering an arbitrary short exact sequence $X=\left(0 \rightarrow X^{\prime} \rightarrow \xi^{\prime} X \rightarrow \xi^{\prime \prime} X^{\prime \prime} \rightarrow 0\right)$ of chain complexes, the maps $\xi^{\prime}, \xi^{\prime \prime}$ being homogeneous of degrees $r^{\prime}, r^{\prime \prime}$, so that $\partial \xi^{\prime}=(-1)^{r^{\prime}} \xi^{\prime} \partial$, $\partial \xi^{\prime \prime}$ $=(-1)^{r^{\prime \prime}} \xi^{\prime \prime} \partial$.

Suppose that $X$ is split by a homomorphism $\phi: X^{\prime \prime} \rightarrow X$ of abelian groups such that $\xi^{\prime \prime} \phi=1: X^{\prime \prime}$. Then $\xi^{\prime \prime}\left(\partial \phi+(-1)^{r \prime \prime} \phi \partial\right)=0$, so that $\partial \phi+(-1)^{r^{\prime \prime}} \phi \partial=\xi^{\prime} \phi^{*}$ where $\phi^{*}: X^{\prime \prime} \rightarrow X^{\prime}$ is a map of degree $-1-r^{\prime}$ $-r^{\prime \prime}$. But $\xi^{\prime}\left(\partial \phi^{*}+(-1)^{r^{\prime}+r^{\prime \prime}} \phi^{*} \partial\right)=0$, so that $\phi^{*}$ is a chain map.

Lemma. If $\phi$ splits $X$ then $H \phi^{*}$ is the homology connecting homomorphism, so that the homology triangle of $\boldsymbol{X}$ is

Received by the editors January 14, 1960. 


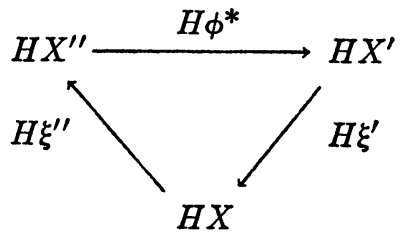

To see this it is only necessary to apply the definition of the homology connecting homomorphism (cf. $[3, \S 10]$ ).

We proceed to the proof of the theorem. The sequence $0 \rightarrow Z X \rightarrow \zeta X$ $\rightarrow \delta B X \rightarrow 0$, where $\zeta$ is the inclusion and $\delta$ is defined by $\zeta \beta \delta=\partial$, $\beta: B X \rightarrow Z X$ being the inclusion, becomes an exact sequence of chain complexes if $Z X$ and $B X$ are provided with zero derivations. Since $X$ is free abelian and $B X$ is a subgroup, $B X$ is also free abelian and the sequence is split, as a sequence of groups, by some $\phi: B X \rightarrow X$.

Tensoring with $Y$ we have

$$
W=(0 \rightarrow Z X \otimes Y \stackrel{\zeta \otimes 1}{\longrightarrow} X \otimes Y \stackrel{\delta \otimes 1}{\longrightarrow} B X \otimes Y \rightarrow 0)
$$

which is exact and is split, as a sequence of groups, by $\phi \otimes(1: Y): B X$ $\otimes Y \rightarrow X \otimes Y$. Now

$$
\begin{aligned}
\partial(\phi \otimes 1)+(\phi \otimes 1) \partial & =\partial \phi \otimes 1-\phi \otimes \partial+\phi \otimes \partial \\
& =(\zeta \otimes 1)(\beta \otimes 1)(\delta \phi \otimes 1)
\end{aligned}
$$

so that $(\phi \times 1)^{*}=\beta \otimes 1: B X \otimes Y \rightarrow Z X \otimes Y$.

Since $B X, Z X$ are free abelian groups the functors $A \rightarrow B X \otimes A$, $A \rightarrow Z X \otimes A$ are exact and $H(B X \otimes Y), H(Z X \otimes Y)$ may be identified with $B X \otimes H Y, Z X \otimes H Y$. Under this identification, $H(\beta \otimes(1: Y))$ becomes $\beta \otimes(1: H Y)$.

We consider, finally, the diagram

$$
0 \rightarrow \text { Tor }_{1}(H X, H Y) \rightarrow B X \otimes H Y \stackrel{\beta \otimes 1}{\rightarrow} Z X \otimes H Y \rightarrow H X \otimes H Y \rightarrow 0
$$

The row is of course exact. By the lemma, the triangle is the homology triangle of $W$ and is thus also exact. Thus maps $\mathfrak{S}: H X \otimes H Y$ $\rightarrow H(X \otimes Y)$ and $\mathfrak{D}: H(X \otimes Y) \rightarrow \operatorname{Tor}_{1}(H X, H Y)$ are defined uniquely by the condition that the augmented diagram commute. The sequence 1.1 is clearly exact. It remains only to observe that all maps 
in the row have degree zero, so that the degrees of $\mathfrak{D}, \mathfrak{S}$ are equal to those of $\delta, \zeta$.

Similar considerations apply when the tensor product is replaced by "Hom." Recall that for $A, B$ graded abelian groups, $\operatorname{Hom}_{q}(A, B)$ $=\prod_{j} \operatorname{Hom}\left(A_{j}, B_{j+q}\right)$. If $X, Y$ are chain complexes the graded group $\operatorname{Hom}(X, Y)$ is provided with a derivation $\tilde{\partial}$ by setting $\tilde{\partial} f=\partial f$ $-(-1)^{q} f \partial$ for $f$ homogeneous of degree $q$. The homology of the resulting chain complex is easily seen to be the group of homotopy classes of chain maps from $X$ to $Y$; we denote it by $\mathfrak{S c o m}(X, Y)$.

Theorem 2. If $X$ and $Y$ are chain complexes and $X$ is free abelian then there is an exact sequence

$$
0 \rightarrow \operatorname{Ext}^{1}(H X, H Y) \stackrel{\mathfrak{D}^{*}}{\longrightarrow} \operatorname{Som}(X, Y) \stackrel{\mathfrak{S}^{*}}{\longrightarrow} \operatorname{Hom}(H X, H Y) \rightarrow 0,
$$

the maps $\mathfrak{D}^{*}, \mathfrak{S}^{*}$ being homogeneous of degrees $-1,0$.

The proof is exactly dual to that of Theorem 1, and may easily be supplied by the reader.

If both $X$ and $Y$ are free abelian then the sequence 1.1 splits, so that $H(X \otimes Y) \approx H X \otimes H Y+\operatorname{Tor}_{1}(H X, H Y)$. We omit the proof of this fact, which will be studied in exhaustive detail in [4].

\section{BIBLIOGRAPHY}

1. H. Cartan and S. Eilenberg, Homological algebra, Princeton, Princeton University Press, 1956.

2. S. Eilenberg and S. MacLane, On the groups $H(\Pi, n)$. II, Ann. of Math. vol. 60 (1954) pp. 49-139.

3. A. Heller, Homological algebra in abelian categories, Ann. of Math. vol. 68 (1958) pp. 484-525.

4. - On the Künneth theorem, to appear.

UNIVERSITY OF ILLINOIS 